\title{
Producción de ácido indol-3-acético por Pseudomonas veronii R4 y formación de raíces en hojas de vid "Thompson seedless" in vitro
}

\author{
Producing Indole-3-Acetic Acid Using Pseudomonas Veronii R4 and in vitro formation of roots in \\ "Thompson Seedless" Grapevine Leaves
}

\author{
María F. Peñafiel-Jaramillo', Emma D. Torres-Navarrete², Alexandra E. Barrera-Álvarez² ${ }^{2}$ Humberto G. Prieto-Encalada ${ }^{3}$, \\ Jaime Morante Carriel $^{4},{ }^{\circ}$ Hayron F. Canchignia-Martínez ${ }^{5}$
}

${ }^{1}$ Facultad de Ciencias Ambientales, Carrera de Gestión Ambiental, Universidad Técnica Estatal de Quevedo. Campus Manuel Haz Álvarez. Av. Quito Km 1.5 vía Santo Domingo de los Tsachilas. EC.120501.Quevedo, Ecuador. mariapenafielj@gmail.com ${ }^{2}$ Facultad de Ciencias Pecuarias, Carrera de Ingeniería Zootécnica e Ingeniería Agropecuaria, Campus Finca Experimental "La Maria” km 7 vía Quevedo-El Empalme. Universidad Técnica Estatal de Quevedo. EC.120501. Quevedo, Ecuador. etorres@uteq.edu.ec; barreraalvarez@yahoo.com;

${ }^{3}$ Laboratorio de Biotecnología, Instituto de Investigaciones Agropecuarias, Estación La Platina. Santa Rosa 11610. La Pintana, Santiago, Chile.Código postal 8831314. hprieto@inia.cl

${ }^{4}$ Facultad de Ciencias Ambientales, Carrera de Ingeniería Forestal, Universidad Técnica Estatal de Quevedo. Campus Manuel Haz Álvarez. Av. Quito Km 1.5 vía Santo Domingo de los Tsachilas. EC.120501.Quevedo, Ecuador. jmorante@uteq.edu.ec ${ }^{5}$ Laboratorio de Microbiología Ambiental y Vegetal, Facultad de Ciencias Agrarias, Escuela de Agronomía, Universidad Técnica Estatal de Quevedo. `hcanchignia@uteq.eud.ec

\section{Resumen}

$\mathrm{L}$ as rizobacterias con actividad antagonista son empleadas a problemas patológicos y promueven el desarrollo de tejido radicular. El objetivo del estudio fue evaluar la capacidad en síntesis de ácido indol-3-acetico (AIA) y la formación de raíces en hojas de vid "Thompson Seedless", mediante el empleo de Pseudomonas veronii R4 in vitro. La bacteria, fue incubada en medio mínimo M9 y se verificó la producción de AIA al muestreo de 24, 48 y 72 h, sobrenadantes fueron analizandos por HPLC. Peciolos de hojas de "Thompson Seedless" fueron sumergidos por $5 \mathrm{~min}$, en el tampón PBS con $P$. veronii R4 a $\left(1.5 \times 10^{9}\right.$ células $\left.\mathrm{mL}^{-1}\right)$, y evaluados a los 32 días postinoculación. Los tratamientos fueron sujetos al análisis de varianza por DCA. Los sobrenadantes muestreados a $72 \mathrm{~h}$, del cultivo de líquido con $P$. veronii $\mathrm{R} 4$, mostraron mayores niveles de AIA entre 0.0231 a $0.0245 \mathrm{mM}$, al emplear 5 a 10 $\mathrm{mM}$ del precursor L-triptofano, del sobrenadante obtenido de P. fluorescens CHA0 la producción de AIA con $0.0174 \mathrm{mM}$ a $10 \mathrm{mM}$ de L-triptofano. Se verificó regeneración del sistema radicular en los peciolos de hojas de "Thompson Seedless", con un promedio de 14 raíces/planta bajo la aplicación de $P$. veronii R4. Estos resultados preliminares realzan la importancia de la cepa nativa $P$. veronii R4, cuyo empleo en condiciones de invernadero para el beneficio en desarrollo del sistema radicular en "Thompson Seedless" se proyecta como objeto de futuras investigaciones.

Palabra claves: sistema radicular, L-triptófano, HPLC, $P$. fluorescens CHA0.

Recibido: 27-diciembre-2015. Recibido en forma corregida: 11-febrero-2016 Aceptado: 12-abril-2016.

Publicado como ARTÍCULO CIENTÍFICO en Ciencia y Tecnología 9(1): 31-36 Junio de 2016
Abstract

D izobacterias having antagonist activity are used to give solution to pathological problems because they promote the development of root tissue in species vegetal. The aim of the study was to evaluate the ability in synthesis of indole-3acetic acid (IAA) and the formation of roots in vine leaves "Thompson Seedless", by the use of Pseudomonas veronii $\mathrm{R} 4$ in vitro. The bacterium was incubated in M9 minimal medium IAA production was verified in samplings at 24, 48 and 72 h., supernatants were analyzed by HPLC. Leaf petioles "Thompson Seedless" were submerged for $5 \mathrm{~min}$ in PBS buffer with $P$. veronii $\mathrm{R} 4\left(1.5 \times 10^{9}\right.$ cells $\left.\mathrm{mL}^{-1}\right)$, evaluated at 32 days post-inoculation. Treatments were subjected to analysis of variance by DCA. The supernatants sampled at $72 \mathrm{~h}$, the culture liquid with $P$. veronii R4, showed higher IAA betweem 0.0231 to $0.0245 \mathrm{mM}$ to employ 5 to $10 \mathrm{mM}$ L-tryptophan precursor, the supernatant obtained from $P$. fluorescens CHA0 production AIA $0.0174 \mathrm{mM}$ to $10 \mathrm{mM} \mathrm{L}$ tryptophan. Regeneration of the root system was verified in leaf petioles "Thompson Seedless", with an average of 14 roots / plant under the application of $P$. veronii $\mathrm{R} 4$. These preliminary results highlight the importance of the native $P$. veronii strain $\mathrm{R} 4$, whose use as greenhouse for the to benefit and development of "Thompson Seedless" root system will be the object for future research.

Key words: root system, L-tryptophan, HPLC, P. fluorescens CHA0. 


\section{Introducción}

$\mathrm{L}$ as especies microbianas pueden alterar el desarrollo del sistema radicular y el crecimiento vegetativo de plantas por la producción de reguladores de crecimiento que incluyen auxinas, etileno y citoquininas (Bowen y Rovira, 1999). Microorganismos aislados de la rizósfera, tienen la capacidad de producir la auxina ácido indol-3-acetico (AIA) in vitro, en presencia o ausencia de precursores fisiológicos como L-triptófano (Trp) (Patten y Glick, 1996). Las fitohormonas como auxina y etileno regulan varios procesos fisiológicos que modifican la arquitectura del sistema radicular, incluyendo la elongación de la raíz primaria (Alonso et al., 2003), la formación de raicillas y elongación (Rahman et al., 2002).

Las células de los ápices meristematicos apicales vegetales producen la auxina AIA y es sintetizada a partir de Trp, aminoácido que se encuentra libre en la planta o formando parte de proteínas (Bialek et al., 1992), aminoácido presente en el meristemo radicular (Taiz y Zeiger, 2006). Las rizobacterias mejoran la proliferación de raíces por la producción de AIA (Asghar et al., 2004; Khalid et al., 2004). La aplicación $P$. fluorescens CHA0 estimula el desarrollo del tejido radicular por producción de auxinas, que en condiciones de cultivo in vitro con el precursor Trp sintetiza AIA (Oberhänsli et al., 1991).

Los trabajos relacionado al empleo de rizobacterias en vides son pocos y resultados en inoculación de $P$. veronii R4 en raíces de "Thompson Seedless", estimula los genes de defensa relacionados con resistencia sistémica inducida (RSI) como: Lox2 y Tlp1 (Peñafiel et al., 2016). Con este antecedente, se procedió a la selección de esta rizobacteria $P$. veronii R4, planteando el objetivo: Evaluar la capacidad de síntesis de AIA in vitro e incremento en el desarrollo de raíces adventicias en hojas de vid "Thompson Seedless".

\section{Materiales y métodos}

\section{Identificación y cuantificación del AIA}

El análisis a síntesis de AIA se realizó en el Laboratorio de Microbiología Molecular y Biotecnología Ambiental de la Universidad Técnica Federico Santa María (UTFSM), Chile. La bacteria en estudio $P$. veronii $\mathrm{R} 4$ y control $P$. fluorescens CHA0, fueron sometidos al análisis de producción de AIA in vitro, con los procedimientos planteados por Suzuki et al. (2003), las bacterias fueron incubados en medio mínimo M9 (g L-1): $\mathrm{Na}_{2} \mathrm{HPO}_{4} 5.8, \mathrm{KH}_{2} \mathrm{PO}_{4} 3, \mathrm{NaCl} 0.5 \mathrm{NH}_{4} \mathrm{Cl} 1$, glucosa 2, $\mathrm{MgSO}_{4}-7 \mathrm{H} 2 \mathrm{O} 0.49, \mathrm{CaCl}_{2}-2 \mathrm{H}_{2} \mathrm{O} 0.0152$, tiamina 0.010, $\mathrm{pH}$ 6.5. Incubados a $28^{\circ} \mathrm{C} / 130 \mathrm{rpm}$. Del cultivo bacteriano se muestreó $700 \mu \mathrm{l}$, el sobrenadante fue centrifugado a 12000 g por $3 \mathrm{~min}$, duplicando el proceso y almacenando a $4{ }^{\circ} \mathrm{C}$. $P$. veronii $\mathrm{R} 4$ y $P$. fluorescens $\mathrm{CHA} 0$ fueron incubados en el medio M9, para su muestreo a: 24, 48 y 72 h., en concentraciones de Trp a $5 \mathrm{mM}$ y $10 \mathrm{mM}$, para evaluación. Con tres replicas experimentales y tres unidades experimentales por muestreo.

Los sobrenadantes se analizaron por Cromatografía liquida de alta presión (HPLC) (Jascoß), en una columna de fase reversa $\mathrm{C} 18\left(5 \mu \mathrm{m} ; 4.6 \times 100 \mathrm{~mm}^{2}\right)$. La fase móvil fue de ácido acético (1.1\%) / acetonitrilo (70:30) con un flujo de $1 \mathrm{~mL}$ min-1. El HPLC fue programado para observar y registrar la absorbancia al máximo punto del espectro de $221 \mathrm{~nm}$, tiempo de retención de $3 \mathrm{~min}$. Se preparó una recta de calibrado de 4 puntos, empleando como control estándar el AIA (Sigma-Aldrich), en concentraciones: 0.0625; 0.25; 0.5 y $1 \mathrm{mM}$. Estándares auténticos de AIA se cuantificaron por integración del área, cada disolución fue inyectada en el HPLC. La linealidad del sistema se realizó por el intervalo de la concentración estudiada, se comprobó mediante un análisis de regresión por el método de mínimos cuadrados.

\section{Interacción entre $P$. veronii $\mathrm{R} 4$ y hojas de "Thompson Seedless"}

El experimento se realizó en el Laboratorio de Biotecnología de La Estación Experimental La Platina del Instituto de Investigaciones Agropecuarias (INIA), Santiago de Chile. Se recolectaron estacas de $15 \mathrm{~cm}$ de longitud con 4 entrenudos, de cultivares de 9 años de la variedad vid 'Thompson Seedless'. Material vegetal tratado con Benomyl $50 \%$ para el control de hongos (Botrytis cinerea), $1 \mathrm{~g} \mathrm{~L}^{-1}$, durante $30 \mathrm{~min}$. Estacas fueron ubicadas en bandejas con agua estéril para emisión de tejido foliar en cámara húmeda a $37^{\circ} \mathrm{C}$ (16 h luz / $8 \mathrm{~h}$ oscuridad) por 40 días, según descrito por McNeilan y Lagerstedt (1967). Las hojas fueron ubicadas en agua estéril para evitar deshidratación y sumergidas en hipoclorito de calcio al $0.1 \%$ por $4 \mathrm{~min}$.

Las rizobacterias se incubaron en $100 \mathrm{~mL}$ de medio liquido King $\mathrm{B}$ hasta alcanzar una concentración de $(1.5 \mathrm{x}$ 109 células $\mathrm{mL}^{-1}$ UFC (Unidades Formadoras de Colonias)), realizando el conteo directo en cámara Neubauer (fondo del campo central de $0.1 \mathrm{~mm}$ y superficie de conteo 0.0025 $\mathrm{mm}^{2}$ ), en microscopio Olympus BX41. Se recuperó $50 \mathrm{~mL}$ del cultivo líquido de $P$. veronii $\mathrm{R} 4$ y $P$. fluorescens $\mathrm{CHA} 0$, centrifugados a $3000 \mathrm{~g}$ por 15 min y lavadas dos veces con un mismo volumen de tampón fosfato salino (PBS) estéril $(\mathrm{pH}$ 6.5) y re-suspendidas las rizobacterias en la misma solución.

La yema y peciolo de hojas de "Thompson Seedless" fueron sumergidas por 5 min en solución PBS con $P$. veronii R4 o $P$. fluorescens CHA0 a la concentración indicada anteriormente. Material vegetal ubicadas en frascos de $9 \mathrm{~cm}$ de largo y 2.5 de diámetro, en la solución sostén agar/agua de $3 \mathrm{~g} \mathrm{~L}^{-1}$ (pH 7) y el control con $0.1 \mathrm{mg} \mathrm{L}^{-1}$ de AIA. Incubación en cámara de crecimiento por 31 días, a $25^{\circ} \mathrm{C}(16 \mathrm{~h}$ luz $/ 8$ $\mathrm{h}$ oscuridad) hasta su evaluación de forma aleatoria. En el tratamiento $P$. veornii $\mathrm{R} 4$ y sus controles $P$. fluorescens $\mathrm{CHA} 0$, $0.1 \mathrm{mg} \mathrm{L}^{-1}$ de AIA, sin inoculante. Sus variables a evaluar fueron 1) porcentaje de enraizamiento $=$ (número de hojas que emitieron raíces x 100 / número total de hojas), 2) número 
de raíces y 3) longitud de raíz mayor. Con cinco replicas experimentales, de tres unidades experimentales (hojas). Los datos generados en síntesis in vitro de AIA y enraizamiento, fueron sujetos a la ANOVA, promedios separados por procedimiento de comparación múltiple de Tukey SD, al nivel de significancia de $(\mathrm{P} \leq 0,05)$, empleando Statgraphics (Statgraphics, 2006).

\section{Resultados y discusión}

\section{Cuantificación de AIA en $P$. veronii $\mathrm{R} 4$}

Los resultados a síntesis de AIA in vitro y adición $5 \mathrm{mM}$ de Trp., mostró mayor incremento para $P$. veronii $\mathrm{R} 4$ y menor producción de la auxina por $P$. fluorescens $\mathrm{CHA} 0$ a los tiempos de muestreo. La producción del AIA in vitro por $P$. veronii $\mathrm{R} 4$ fue de $0.0166 \mathrm{mM}$ a $24 \mathrm{~h}$, síntesis que se mantiene entre $0.0231 \mathrm{mM}$ de $48 \mathrm{~h}$ hasta $72 \mathrm{~h}$ de muestreo, no generando incremento en producción de auxina. Por lo contario en $P$. fluorescens CHA0 la producción de AIA fue de $0.0011 \mathrm{mM}$ a $72 \mathrm{~h}$ de muestreo (Figura 1A).
El aumento del precursor de $10 \mathrm{mM}$ de Trp. en el medio de cultivo M9 con $P$. veronii $\mathrm{R} 4$, no genera aumento en la síntesis en AIA de $0.0160 \mathrm{mM}$ a $24 \mathrm{~h}$, síntesis que se mantiene entre $0.0249 \mathrm{mM}$ y $0.0251 \mathrm{mM}$ de AIA, para los tiempos de muestreo de 48 a $72 \mathrm{~h}$ respectivamente. Por el contrario, la cepa control $P$. fluorescens $\mathrm{CHA} 0$ en presencia de $10 \mathrm{mM}$ de Trp., llega a niveles de síntesis en AIA de 0.011 hasta 0.0174 $\mathrm{mM}$ a 24 a $72 \mathrm{~h}$ (Figura 1B).

La producción de AIA por ambos microorganismo fue estimulada por la adición de Trp en el medio de cultivo M9. La cepa $P$. veronii $\mathrm{R} 4$ en presencia de Trp a $10 \mathrm{mM}$, alcanza la mayor síntesis en AIA de $0.0251 \mathrm{mM}$. A diferencia de los obtenidos por Valérie et al. (2007), que registraron menores niveles de producción en AIA de $0.018 \mathrm{mM}$, con Trp $(200 \mu \mathrm{g} /$ $\mathrm{mL}^{-1}$ ) in vitro a $72 \mathrm{~h}$ de muestreó, en Pseudomonas putida.

La diferencia en síntesis del AIA por Pseudomonas spp, depende al empleo del precursor, los resultados obtenidos por $P$. veronii $\mathrm{R} 4$ se observan niveles de producción en AIA de $0.0251 \mathrm{mM}$ con Trp. A diferencia de los obtenidos por Valérie et al. (2007), al cambiar el tipo de precursor L-triptófano por triptamina, incrementan la síntesis en AIA de 0.018 a 0.133

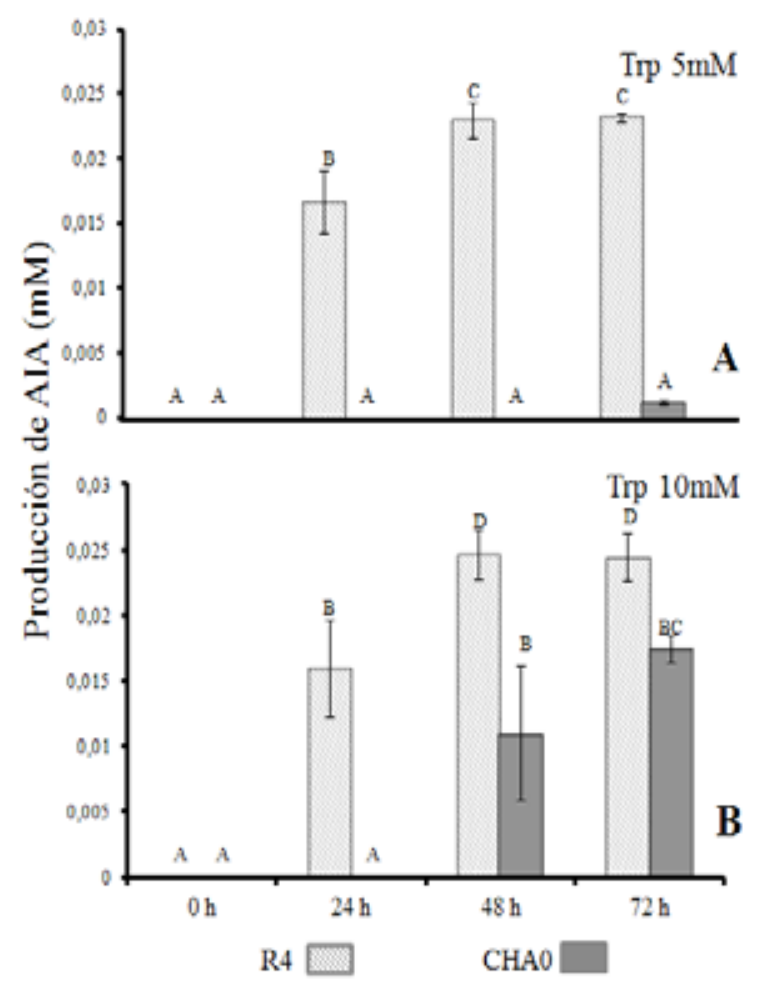

Figura 1. Síntesis de AIA en cepas $P$. veronii $\mathrm{R} 4$ y $P$. fluorescens CHA0, a diferentes concentraciones de $\operatorname{Trp}(\mathrm{A}$ y B). Producción in vitro de AIA por $P$. veronii $\mathrm{R} 4$ y $P$. fluorescens CHA0, en adición de Trp a 5 mM y 10 mM, muestreo de 24, 48 y 72 h. Los valores con letras similares no representan diferencias estadísticas significativas al nivel de $(\mathrm{P} \leq \mathbf{0 , 0 5})$, por el procedimiento de comparación múltiple de Tukey. Las barras de error indican la desviación estándar (DS) 
$\mathrm{mM}$, en Pseudomonas putida. El incremento del AIA por la aplicación de triptamina, corresponde a una de las cuatro vías metabólicas dependientes de Trp (Bartel, 1997). Esto sugiere la existencia de la ruta metabólica del Trp, pero no se puede definir cuál de las cuatro rutas metabólicas (triptamina, triptofol, ácido indol-3-pirúvico, vía del indol-3-acetamida) posee $P$. veronii $\mathrm{R} 4$, en la síntesis de AIA.

La incubación de $P$. veronii $\mathrm{R} 4$ en el medio de cultivo M9 y el aumento del precursor Trp a $10 \mathrm{mM}$, no se verifica incremento en síntesis de AIA entre 0.0251 y $0.0231 \mathrm{mM}$, en muestreos de 48 a $72 \mathrm{~h}$ respectivamente. Estos resultados coinciden con Sridevi y Mallaiah (2007), en Rhizobium spp 6 no incrementa en la síntesis de AIA entre 0.068 y $0.070 \mathrm{mM}$, para los mismos tiempos de muestreo. Además, el incremento de la dosis del precursor Trp no mejora la capacidad productora del AIA por P. veronii R4, por efecto de la liberación de enzimas de degradación como peroxidasa y oxidasa del AIA, reportado en Rhizobium spp (Datta y Basu, 2000). En este estudio se considera que $P$. veronii R4 es capaz de degradar parcialmente el AIA in vitro, lo que se estaría comprobando que tienen similares respuesta a $P$. putida 1290 , capaz de utilizar el AIA como su única fuente de energía en medio M9 (Glick et al., 1998).

La síntesis de AIA de $0.0251 \mathrm{mM} \mathrm{a} 72 \mathrm{~h}$ de muestreo por P. veronii $\mathrm{R} 4$ son menores, al comparar con $P$. putida GR122 , incrementa su producción de AIA en $(0.082 ; 0.128 ; 0.149$; $0.186 \mathrm{mM})$ por el aumento del Trp entre $(50 ; 100 ; 200 ; 500$ $\mu \mathrm{g} \mathrm{mL}^{-1}$ ) respectivamente (Patten y Glik, 2002). La diferencia en producción estaría regulada por la ruta metabólica del AIA y la presencia de enzimas degradación. La síntesis de AIA in vitro en $P$. fluorescens CHA0 (Figura 1B), fueron similares con los obtenidos por Oberhänsli et al. (1991), en producción de $0.02 \mathrm{mM}$ en AIA a $72 \mathrm{~h}$ de muestreo. Los resultados observables por $P$. fluorescens $\mathrm{CHA} 0$, valida el procedimiento experimental en la síntesis de AIA in vitro.

\section{Emisión de raíces en hojas de 'Thompson Seedless'}

Las exposición directa de las hojas de 'Thompson Seedless' por $P$. veronii R4 y los controles $P$. fluorescens CHA0 y AIA $\left(0.1 \mathrm{mg} \mathrm{L}^{-1}\right)$ y sin bacteria, mostraron efecto en producción de sistema radicular. La aplicación $P$. veronii R4 genera entre 14 raíces / hoja, con abundante producción de raíces laterales. Tejido radicular que se distribuye alrededor de yema y peciolo de la hoja, caso contrario en los controles $P$. fluorescens $\mathrm{CHA} 0$ y AIA $\left(0.1 \mathrm{mg} \mathrm{L}^{-1}\right)$ disminuyeron a 3 raíces / hoja, con un patrón preferente al final de la yema (Figura 2A).

La aplicación de $P$. veronii R4 incrementa la formación de tejido radicular en hojas, resultados que permite confirma su aplicación como PGPR en vid 'Thompson Seedless'. Esto concuerda con Bent et al. (2001), con aplicaciones P. fluorescens M20 en plántulas in vitro de Pinus contorta Dougl., favorecen al aumento del sistema radicular. Además, la aplicación in vitro de Burkholderia phytofirmans PsJN en
Vitis vinifera L. cv. Chardonnay clon 7535, incrementa la masa radicular por la colonización endofítica (Barka et al., 2006).

Las hojas de vid 'Thompson Seedless' y P. veronii R4 establece una relación simbiótica, la rizobacteria capta las pequeñas cantidades de Trp presente en sus primeras raíces, incrementando la síntesis de AIA que estimulan el desarrollo del sistema radicular. Por otro lado Kravchenko et al. (2004), manifiestan que las bacterias en asociación con la planta captan el Trp del producto de degradación en raíces y exudados radiculares, produciendo el aumento AIA. Woodward y Bartel (2005), indican que el AIA es la principal auxina en especies vegetales, controlando muchos procesos fisiológicos: La división y alargamiento celular, diferenciación de tejidos.

En longitud del sistema radicular, por aplicación del control P. fluorescens CHA0 genera raíces de $4.4 \mathrm{~cm}$ en hojas de 'Thompson Seedless'. La aplicación de P. veronii R4, los controles AIA y sin bacteria fueron similares, obteniendo longitudes promedios de raíces $1.5,1.4$ y $0.9 \mathrm{~cm}$, respectivamente (Figura $2 \mathrm{~B}$ ). Las altas concentraciones de AIA inciden en disminución en longitud del sistema radicular. Glick et al. (1999), explican que bacterias que secretan bajos niveles de AIA (10-9 a 10-12 mM), estimulan la elongación de raíces, mientras que bacterias altamente productoras de auxinas promueven la formación de raíces laterales o el desarrollo de pelos absorbentes.

En porcentaje de enraizamiento en hojas de 'Thompson Seedless', la aplicación individual de $P$. veronii $\mathrm{R} 4$ y $P$. fluorescens $\mathrm{CHA} 0$, se obtiene el mayor porcentaje de emisión del sistema radicular con 96 y 88\%. La aplicación de los controles AIA $\left(0.1 \mathrm{mg} \mathrm{L}^{-1}\right)$ y sin inoculante se observa la disminución del enraizamiento con 68 y $64 \%$ en hojas de 'Thompson Seedless' (Figura 2C). Las hojas de 'Thompson Seedless', en los controles sin bacteria se observa la producción basal de raíces con menor tamaño y número. Las plantas son capaces de sintetizar AIA en los ápices meristemáticos de tejido verde (tallos, primordios de hoja y hojas jóvenes) a partir del precursor Trp (Ljung et al., 2001).

Las hojas de vid 'Thompson Seedless' tratadas con $P$. veronii $\mathrm{R} 4$ y $P$. fluorescens $\mathrm{CHA} 0$, mantuvieron un color verde intenso. Las rizobacterias reducen la producción de etileno en la planta, inhibiendo la estimulación de senescencia y estrés. Las bacterias poseen la enzima deaminasa aminociclopropano carboxilato (ACC), que degrada al ACC, precursor del etileno (Glick et al., 2007). Enzima deaminasa ACC, son capaces de reducir los niveles endógenos de etileno en la planta por efecto de las rizobacterias (Glick et al., 1998). El etileno es una hormona vegetal producida bajo estrés, que en altas concentraciones inhibe el crecimiento vegetal. La reducción en los niveles de etileno por acción de las rizobacterias promotoras del crecimiento, generando un mayor desarrollo por plantas inoculadas (Husen et al., 2011). 

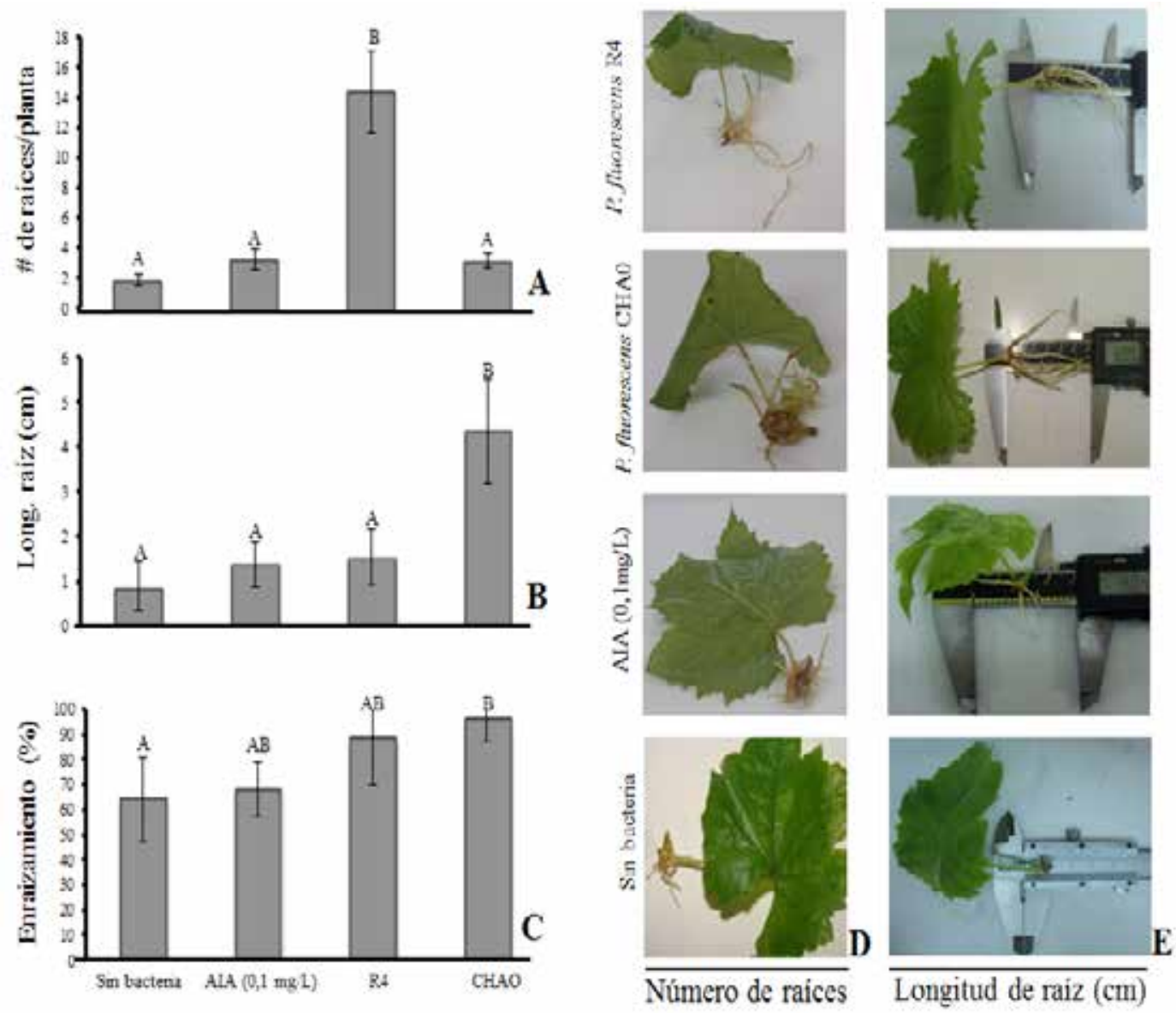

Figura 2. Efecto de $P$. veronii $R 4$ y $P$. fluorescens CHA0 sobre el enraizamiento en hojas de 'Thompson Seedless'. A, Número de raíces. B, Longitud de raíz mayor en cm. C, Porcentaje de enraizamiento, evaluados a 32 días. D y E, Formación de tejido radicular y longitud de la raíz en hojas de 'Thompson Seedless'. Los valores con letras similares no representan diferencias estadísticas significativas al nivel de $(\mathrm{P} \leq \mathbf{0 , 0 5})$, por el procedimiento de comparación múltiple de Tukey. Las barras de error indican la desviación estándar (DS).

\section{Conclusión}

T os niveles en producción de AIA in vitro no superan el $0.0251 \mathrm{mM}$ a las $72 \mathrm{~h}$ de muestreo, por la presencia de enzimas digestivas que se están produciendo en el medio de cultivo, empleando la auxina como fuente de energía. Es necesario indagar al empleo del precursor para determinar el modelo de síntesis de AIA y verificar que ruta metabólica estaría participando para el incremento de la auxina. Al promedio longitud del sistema radicular en hojas de 'Thompson Seedless', la aplicación de $P$. veronii R4 estaría involucrado en el proceso de degradación del AIA y reducir el efecto por altas concentraciones de la hormona. Estos resultados preliminares realzan la importancia de la cepa nativa $P$. veronii $\mathrm{R} 4$, que hace factible su estudio en condición de invernadero para determinar el incremento del sistema radicular.

\section{Agradecimiento}

Beca CONICYT otorgado del 2008 al 2012. Valparaíso-Chile. Doctorado en Biotecnología de la Pontifica Universidad Católica de Valparaíso y Universidad Técnica Federico Santa María. Trabajo financiado por Biofrutales S.A. Consorcio y la CORFO-Chile otorgado 13CTI-21520-SP07. "Nuevas tecnologías de mejoramiento Genético especies frutales, desarrollo de nuevos cultivares de Vides y Frutales de Carozos tolerantes a hongos y virus".

\section{Bibliografía}

Alonso, M., Stepanova, N., Solano, R., Wisman, E., Ferrari, S., Ausubel, M. and Ecker, R. 2003. Five components of the ethylene-response pathway identified in a screen for weak ethylene-insensitive mutants in Arabidopsis. 
Proceedings National Academy Sciences. U.S.A. 100: 2992-2997.

Asghar, H., Zahir, Z. and Arshad, M. 2004. Screening rhizobacteria for improving the growth, yield and oil content of canola (Brassica napus L.). Australian Journal Agricultural Research. 55: 187-194.

Barka, E., Nowak, J. and Clément, C. 2006. Enhancement of chilling resistance of inoculated grapevine plantlets with a plant growth-promoting rhizobacterium, Burkholderia phytofirmans Strain PsJN. Applied and Environmental Microbiology. 72: 7246-7252.

Bartel, B. 1997. Auxin biosynthesis. Annual Review of Plant Physiology and Plant Molecular Biology. 48: 51-66.

Bent, E., Tuzun, S., Chanway, C. and Enebak, S. 2001. Alterations in plant growth and in root hormone levels of lodgepole pines inoculated with rizobacteria. Canadian Journal of Microbiology. 47: 793-800.

Bialek. K., Michalczuk. L. and Cohen, J. 1992. Auxin biosynthesi during seed germination in Phaseolus vulgaris. Plant Physiology. 100: 509-517

Bowen, D. and Rovira, D. 1999. The rhizosphere and its management to improve plant growth. Advances in Agronomy. 66:1-102.

Datta, C. and Basu, P. 2000. Indole acetic acid production by a Rhizobium species from root nodules of a leguminous shrub, Cajanus cajan. Microbiology Research. 155: 123127.

Husen, E., Wahyudi, A. and Gianto, A. 2011. Soybean response to 1-aminociclopropano-1-carboxylate deaminaseproducing Pseudomonas under fild soil conditions. American Journal of Agricultural and Biological Sciencies. 6:273-278.

Glick, B., Penrose, D. and Li, J. 1998. A model for the lowering of plant ethylene concentrations by plant growth-promoting bacteria. Journal of Theoretical Biology. 190: 63-68.

Glick, B., Patten, C., Holguin, G. and Penrose, D. 1999. Biochemical and genetic mechanisms used by plant growth promoting bacteria. Imperial College press. London. $270 \mathrm{p}$.

Glick, B., Cheng, Z., Czarny, J. and Duan, J. 2007. Promotion of plant growth by ACC deaminase-producing soil bacteria. European Journal of Plant Pathology. 119:329339.

Khalid, A., Arshad, M., and Zhair, Z. 2004. Screening plant growth-promoting rhizobacteria for improving growth and yield of wheat. Journal Applied Microbiology. 96: 473-480.

Kravchenko, L., Azarova, T., Makarova, M. and Tikhonovich, I. 2004. Theeffect of tryptophan present in plant root exudates on the phytostimulating activity of rhizobacteria. Microbiology. 73: 156-158.
Ljung, K., Rishikesh. P., Bhalerao. D. and Sandberg, G. 2001 Sites and homeostatic control of auxin biosynthesis in Arabidopsis during vegetative growth. Theplantjournal. 28: 465-474.

McNeilan, R. and Lagerstedt, H. 1967. Plant propagation by leaf and leaf-bud cuttings. Assistant Professor of Horticulture. 115-116.

Oberhänsli, T., Défago, G. and Haas, D. 1991. Indole-3-acetic acid (IAA) synthesis in the biocontrol strain CHAO of Pseudomonas fluorescens: role of tryptophan side chain oxidase. Journal of General Microbiology. 137: 22732279 .

Patten, C. and Glick, B. 1996. Bacterial biosynthesis of indole-3-acetic acid. Canadian Journal of Microbiology. 42: 207-220.

Patten, C. and Glick, B. 2002. Role of Pseudomonas putida indoleacetic acid in development of the host plant root system. Applied and Environmental Microbiology. 68: 3795-3801.

Peñafiel, M., Sánchez. E., Cruz, N., Belezaca, C., Prieto, H. y Canchignia, H. 2016. Activación de resistencia sistémica inducida en vid "Thompson Seedless", en respuesta Pseudomonas veronii R4. Ciencia y Tecnología. 9(1):19.

Rahman, A., Hosokawa, S., Oono, Y., Amakawa, T., Goto, N. and Tsurumi, S. 2002. Auxin and ethylene response interactions during Arabidopsis root hair development dissected by auxin influx modulators. Plant Physiology. 130:1908-1917.

Suzuki, S., Yuxi, H. and Oyaizu, H. 2003. Indole-3-acetic acid production in Pseudomonas fluorescens HP72 and its association with suppression of creeping bentgrass brown patch. Current Microbiology. 47: 138-143.

Sridevi, M. and Mallaiah, K. 2007. Bioproduction of indole acetic acid by Rhizobium strains isolated from root nodules of green manure crop, Sesbania sesban (L.) Merr. Iranian Journal of Biotechnology. 5: 178-182.

Statgraphics. 2006. Statgraphics Centurion XV. Stat.Point. Assuring excellence. Disponible en www.statpoint.com.

Taiz, L. and Zeiger, C. 2006. Auxin: The growth hormone. En: Plant Physiology. Sinauer Associates. Inc. Publishers (4 Ed). Sunderland, Massachusetts, USA. 467-507.

Valérie, G., Hani, A. and Russell, J. 2007. Growth stimulation and fruit yield improvement of greenhouse tomatoplants by inoculation with Pseudomonas putida or Trichodermab atroviride: Possible role of indole acetic acid (IAA). Soil Biology \& Biochemistry. 39: 1968-1977.

Woodward. A. and Bartel, B. 2005. Auxin: Regulation, Action, and Interaction. Annals of Botany. 95: 707-735. 